\title{
COMPARISON OF BONE PARAMETERS BY BODY REGION IN UNIVERSITY ATHLETES: SYSTEMATIC REVIEW
}

\author{
COMPARAÇÃO DOS PARÂMETROS ÓSSEOS POR REGIÃO CORPORAL EM ATLETAS UNIVERSITÁRIOS: \\ REVISÃO SISTEMÁTICA
}
COMPARACIÓN DE PARÁMETROS ÓSEOS POR REGIÓN CORPORAL EN ATLETAS UNIVERSITARIOS: REVISIÓN SISTEMÁTICA

Mikael Seabra Moraes' (iD) (Physical Education Professional) Priscila Custódio Martins' (ID (Physical Education Professional) Diego Augusto Santos Silva' (iD (Physical Education Professional)

1. Federal University of Santa Catarina, Research Center in Kinanthropometry and Human Performance, Florianópolis, Santa Catarina, Brazil.

\section{Correspondence}

Diego Augusto Santos Silva. Universidade Federal de Santa Catarina - Centro de Desportos, Departamento de Educação Física, Campus Universitário - Trindade - Caixa Postal 476, CEP 88040-900. Florianópolis, Santa Catarina, Brazil. diegoaugustoss@yahoo.com.br

\begin{abstract}
Introduction: Bone mineral density (BMD) and bone mineral content (BMC) vary depending on the type of sport practiced and the body region, and their measurement can be an effective way to predict health risks throughout an athlete's life. Objective: To describe the methodological aspects (measurement of bone parameters, body regions, precision errors and covariates) and to compare BMD and BMC by body region (total body, upper limbs, lower limbs and trunk) among university athletes practicing different sports. Methods: A search was performed on the databases PubMed, Web of Science, Scopus, ScienceDirect, EBSCOhost, SportDiscus, LILACS and SciELO. Studies were selected that: (1) compared BMD and BMC of athletes practicing at least two different sports (2) used dual-energy X-ray absorptiometry (DXA) to assess bone parameters (3) focused on university athletes. The extracted data were: place of study, participant selection, participants'sex, sport practiced, type of study, bone parameters, DXA model, software used, scan and body regions, precision error, precision protocol, covariates and comparison of bone parameters between different sports by body region. Results: The main results were: 1) BMD is the most investigated bone parameter; 2 ) total body, lumbar spine and proximal femur (mainly femoral neck) are the most studied body regions; 3) although not recommended, the coefficient of variation is the main indicator of precision error; 4) total body mass and height are the most commonly used covariates; 5) swimmers and runners have lower BMD and BMC values; and 6) it is speculated that basketball players and gymnasts have greater osteogenic potential. Conclusions: Swimmers and runners should include weight-bearing exercises in their training routines. In addition to body mass and height, other covariates are important. The results of this review can help guide intervention strategies focused on preventing diseases and health problems during and after the athletic career. Level of evidence Il; Systematic Review.
\end{abstract}

Keywords: Absorptiometry, photon; Bone and Bones; Bone remodeling; Athletic performance; Universities.

\section{RESUMO}

Introdução: A densidade mineral óssea (DMO) e o conteúdo mineral ósseo (CMO) variam dependendo do esporte praticado e região corporal, e sua medição pode ser uma forma eficaz de prever riscos para a saúde ao longo da vida de um atleta. Objetivo: Descrever os aspectos metodológicos (mensuração dos parâmetros ósseos, regiões corporais, erros de precisão e covariáveis) e comparar a DMO e o CMO por região corporal (corpo total, membros superiores, membros inferiores e tronco) em atletas universitários de diferentes modalidades esportivas. Métodos: A busca foi realizada nos bancos de dados PubMed, Web of Science, Scopus, ScienceDirect, EBSCOhost, SportDiscus, LILACS e SCiELO. Foram selecionados estudos que: (1) compararam a DMO e o CMO de atletas que praticam pelo menos dois esportes; (2) usaram absorciometria de raios X de dupla energia (DXA) para avaliaros parâmetros ósseos e (3) com foco em atletas universitários. Os dados extraídos foram local do estudo, seleção dos participantes, sexo dos participantes, esporte praticado, tipo de estudo, parâmetros ósseos, modelo DXA software utilizado, varredura e regiões corporais, erro de precisão, protocolo de precisão, covariáveis e comparação de parâmetros ósseos entre esportes por região do corpo. Resultados: Os principais resultados foram: 1) DMO como parâmetro ósseo mais investigado; 2) corpo total, coluna lombar e parte proximal do fêmur (principalmente colo do fêmur) como as regiões corporais mais estudadas; 3) embora não seja recomendado, o coeficiente de variação foi o principal indicador de erro de precisão; 4) massa corporal total e estatura como covariáveis mais usadas; 5) nadadores e corredores têm valores mais baixos de DMO e CMO e 6) especula-se quejogadores de basquete e ginastas têm maior potencial osteogênico. Conclusões: Nadadores e corredores devem incluir exercícios de sustentação de peso na rotina de treinamento. Além da massa corporal e da estatura, outras covariáveis são importantes. Os resultados desta revisão podem ajudar a orientar estratégias de intervenção focadas na prevenção de doenças e problemas de saúde durante e depois da carreira esportiva. Nível de evidência II, Revisão sistemática.

Descritores: Absorciometria de fóton; Osso e Ossos; Remodelação óssea; Desempenho atlético; Universidades. 


\section{RESUMEN}

Introducción: La densidad mineral ósea (DMO) y el contenido mineral óseo (CMO) varían en función del deporte practicado y de la región corporal, y su medición puede ser una forma efectiva de predecir los riesgos para la salud a lo largo de la vida de un atleta. Objetivo: Describir los aspectos metodológicos (medición de parámetros óseos, regiones corporales, errores de precisión y covariables) y comparar la DMO y el CMO por región corporal (cuerpo total, miembros superiores, miembros inferiores y tronco) en atletas universitarios de diferentes deportes. Métodos: La búsqueda se realizó en las bases de datos PubMed, Web of Science, Scopus, ScienceDirect, EBSCOhost, SportDiscus, LILACS y SciELO. Se seleccionaron estudios que: (1) compararon la DMO y el CMO de atletas que practicaban al menos dos deportes; (2) utilizaron la absorciometría de rayos X de doble energía (DXA) para evaluar los parámetros óseos y (3) se centraron en atletas universitarios. Los datos extraídos fueron: ubicación del estudio, selección de los participantes, sexo de los participantes, deporte practicado, tipo de estudio, parámetros óseos, modelo de DXA, software utilizado, escaneo y regiones corporales, error de precisión, protocolo de precisión, covariables y comparación de parámetros óseos entre deportes por región corporal. Resultados: Los principales resultados fueron: 1) DMO como el parámetro óseo más investigado; 2) cuerpo total, columna lumbar y parte proximal del fémur (principalmente cuello femoral) como las regiones corporales más estudiadas; 3) aunque no se recomienda, el coeficiente de variación fue el principal indicador de error de precisión; 4) la masa corporal total y la altura fueron las covariables más utilizadas; 5) los nadadores y corredores presentan valores más bajos de DMO y $\mathrm{CMO}$;6) se especula un mayor potencial osteogénico en jugadores del baloncesto y gimnastas. Conclusiones: Los nadadores y corredores deben incluir ejercicios con pesas en su rutina de entrenamiento. Además de la masa corporal y la altura, otras covariables son importantes. Los resultados de esta revisión pueden guiar las estrategias de intervención centradas en la prevención de enfermedades y problemas de salud durante y después de la carrera deportiva. Nivel de evidencia II, Revisión Sistemática.

Descriptores: Absorciometría de fotón; Huesos; Remodelación ósea; Rendimiento atlético; Universidades.

\section{INTRODUCTION}

Bone is living tissue with vital functions such as structural and mineral storage.' The effect of sports participation on bone parameters during childhood and adolescence ${ }^{2-6}$ and young adulthood ${ }^{3,7-9}$ has been widely described. These surveys identified that individuals who practiced sports during the first decades of life significantly reduced the risk of morbidity and mortality caused by diseases resulting from the deterioration of bone structures, such as osteoporosis, osteoarthritis and fractures. Two macro bone health indicators are bone mineral content (BMC) and bone mineral density (BMD). ${ }^{10}$

In the sporting context, the adaptive responses that directly impact BMD and BMC vary according to the magnitude, speed and frequency of the activity load. ${ }^{9,11-13}$ Nikander et al. ${ }^{12}$ suggest an interesting classification of the osteogenic effect of sports: (1) high-magnitude vertical impacts (gymnastics), (2) moderate-magnitude impacts from varying, unusual directions (soccer and badminton), (3) high-magnitude muscle forces (powerlifting), (4) a great number of consecutive low-to-moderate-magnitude impacts (long distance running), and (5) a great number of consecutive non-weight-bearing muscle contractions (swimming). ${ }^{12,13}$

Although the direction regarding the comparison between sports has already been established, that is, the greater the impact loading of the sport the more osteogenic it is, ${ }_{1}^{11,12,14}$ has not yet been summarized in the literature, the comparison between sports in specific body regions, as upper limbs, lower limbs and trunk. This comparison can differentiate $\mathrm{BMD}$ and BMC from sports that belong to the same impact classification and that have an emphasis on the same in the body region.

Additionally, other factors can affect bone health in athletes such as inadequate nutrition, not practicing weight-bearing exercise, calcium and vitamin D, menstrual irregularities, use of drugs and hormones ${ }^{8,9}$ Therefore, it is necessary to consider them when analyzing the impact of sport on bone parameters. In addition, dual-energy $x$-ray absorptiometry (BMD) is the reference method for BMD assessment, but the precision error must follow a strict protocol. ${ }^{15-17}$ The International Society for Clinical Densitometry (ISCD) strongly recommends that the precision error be calculated to determine the least significant change (LSC), which is the least amount of change in BMD that can be considered statistically significant. 16,17 The detailed protocol for athletes was describe, ${ }^{16}$ but no systematic review study summarized this information, which was done in the present review.

College athletes will be the focus of this review, since approximately $20 \%$ of bone mass can be modified until the third decade of life, ${ }^{8}$ that is, these athletes are more susceptible to changes in bone metabolism by modifiable factors than professional athletes, which can assist in future interventions focused on athletes' bone health. In addition, university athletes generally have less training and years of practice than professional athletes, ${ }^{18}$ which sets them apart for health outcomes. Therefore, the aim of this review was to describe the methodological aspects (bone parameters measured by DXA, investigated body regions, precision errors and covariates) and to compare BMD and BMC by body region (total body, upper limbs, lower limbs and trunk) among university athletes from different sports.

\section{METHOD}

\section{Records}

The protocol of this systematic review was recorded in International Prospective Register of Systematic Reviews (PROSPERO), with registration number CRD42018083113. The report of this review is in agreement with the Preferred Reporting Items for Systematic Reviews (PRISMA). ${ }^{19}$

\section{Sources of information}

The systematic search was performed until January 13, 2021 in eight databases: 1) Medical Literature Analysis and Retrieval System Online (MEDLINE), through PubMed; 2) Web of Science; 3) Scopus; 4) ScienceDirect; 5) MEDLINE, through the EBSCOhost platform; 6) SportDiscus; 7) Latin American and Caribbean Literature in Health Sciences (LILACS); and 8) Scientific Electronic Library Online (SciELO).

\section{Search strategy, descriptors and keywords}

The search for articles in the databases was carried out by groups of descriptors inserted in the Medical Subject Headings (MeSH) platform 
and by keywords selected by consensus from published scientific sources. Depending on the database, these terms were entered in English and/or Portuguese. The groups of descriptors and keywords were: 1) result (bone parameters); 2) instrument (DXA); 3) population (university athlete). The definition of terms and tests in the databases was carried out by two independent researchers (MSM and PCM). More details are described in supplementary table $1 .{ }^{20}$

\section{Data availability}

Every data set that supports the results of this study was provided to the Scielo Dataverse repository and can be accessed at https://doi. org/10.48331/scielodata.R9NN4H. ${ }^{20}$

\section{Eligibility criteria}

The following inclusion criteria were used: a) original articles (all types); b) articles that compared bone parameters (BMC and BMD), as primary or secondary outcomes, at least between two sports; $\mathrm{c}$ ) studies that used DXA to measure BMD and BMC in any region of the body; d) samples with university athletes; e) all studies published until January 2021.

The exclusion criteria were: a) review articles (all types), theses, dissertations, abstracts published in annals of scientific congresses, opinion articles; b) studies with athletes outside the established age range; c) studies with elite, amateur, professional and leisure athletes; d) studies that used other non-DXA techniques to estimate BMD and BMC; d) articles that made comparisons between athletes and non-athletes; e) articles not available and that no response was obtained from the authors via email and the research portal, after two attempts.

\section{Selection of studies}

Two independent reviewers examined each database (MSM and PCM) for potential articles. After extracting the articles from the databases, the exclusion criteria were applied to duplicate articles, reading titles and abstracts and reading selected articles in full. In addition, references for each selected study were read to identify other potential articles. Disagreements between reviewers were resolved by consensus. The opinion of a third reviewer (DASS) was consulted in the event of an unresolved disagreement.

The Zotero ${ }^{\circledR}$ version 5.0 bibliographic manager (Roy Rosenzweig Center for History and New Media, Fairfax, Virginia, USA) was used to create specific libraries, identification and exclusion of duplicate studies, division and organization of results of each database.

\section{Data extraction}

Data extraction was inserted into tables and figures grouped into two categories: (a) descriptive characteristics of studies - author, place of study, sex, sports, type of study, DXA model (manufacturer, state, country), software used and bone parameters investigated (BMD or BMC); (b) comparison of bone parameters between sports by body region and covariates that interfere in the bone-sport relationship. The covariates were summarized in two ways: 1) all covariates tested in the articles; 2) covariates used in the final statistical model. Additional information on the articles (selection of participants, comparator group, scan and body regions, precision error and precision protocol) was summarized in supplementary table $2^{20}$. Data extraction was performed by two independent researchers (MSM and PCM).

\section{Risk of bias}

The evaluation of the risk of bias of included studies was performed by two independent researchers (MSM and PCM). In case of disagreement, the opinion of a third researcher (DASS) was requested. The Quality Assessment Tool for Observational Cohort and Cross-Sectional Studies proposed by the National Heart, Lung and Blood Institute $(\mathrm{NIH})^{21}$ was used to assess the risk of bias.
For each evaluated criterion, "yes", "no", "cannot be determined", "not applicable" or "not reported" were assigned. Based on the number of positive responses, the final score was calculated by dividing the number of "yes" answers by the total tool criteria. In this calculation, the criterion with "cannot be determined" or "not reported" responses negatively counted and the "not applicable" criterion were excluded from the calculation to determine the final risk of bias score of studies. The study was classified as low risk of bias (final score $\geq 0.70$ ), moderate risk of bias (final score $<0.70$ ) or high risk of bias (final score $<0.50$ ). ${ }^{22}$

\section{RESULTS}

A total of 2,776 records, excluding duplicates, was identified in this systematic review. After reading titles and abstracts, 123 articles (4.43\%) were evaluated by full reading. Of these, 105 articles were excluded because they did not meet the eligibility criteria, totaling 18 articles $(0.65 \%)$. One article was added after the reading of references, which resulted in 19 articles (ten cross-sectional, seven longitudinal and two mixed studies) included in the review. (Figure 1 and Table 1)

\section{Place of study and sex of participants}

Of the 19 articles included in the review, 18 were conducted in the United States (USA) and one was conducted in Japan. In relation to gender, 14 articles proposed to study the BMD and/or BMC only in female athletes, one study only in male athletes and four studies in athletes of both sexes. (Table 1)

\section{Sports and comparator group}

Thirteen sports were identified in the articles included in the review, with eight individual sports (swimming, gymnastics, tennis court, athletics, judo, rowing, wrestlers and golf) and six team sports (volleyball, basketball, softball, field hockey, soccer and football). One article categorized sports into lean-build sports and non-lean build sports. (Table 1)

With 11 articles, athletics (without distinguishing the type of athletics competition) was the most investigated sport. In sequence, the sports most investigated were swimming ( $n=10$ articles) and basketball ( $n=08$ articles). The comparator group of all studies was the sports investigated. (Table 1) More details of comparator group and selection of participants can be seen in the Supplementary Table $2^{20}$.

\section{DXA model and bone parameters}

Most studies reported used Hologic ${ }^{\circledR}$ DXA models (Wisconsin, USA) (nine articles) and Lunar ${ }^{\circledast}$ (Massachusetts, USA) (nine articles). One article reported using the Norland ${ }^{\circledR}$ model (Wisconsin, USA). (Table 1) Regarding bone parameters, 15 articles evaluated only BMD, one article evaluated only BMC and three articles evaluated BMD and BMC together. (Table 1)

\section{Body regions analyzed}

With 15 articles, the total body was the most investigated body region. In addition, the anteroposterior lumbar spine was reported in 10 articles (six articles from $L 2$ to $L 4$ and four articles from $L 1$ to $L 4$ ). Additionally, the highlight on the upper limbs were the arms with six articles and on the lower limbs, the highlights were the femoral neck with nine articles and the legs with eigth articles. In addition, in the trunk region, the pelvis was the highlight with six articles. (Figure 2)

\section{Precision error and protocol used}

Twelve articles expressed precision errors using the $\%$ CV , one article expressed $\mathrm{g} / \mathrm{cm}^{2}$ (reported by the manufacturer) and six did not report the precision error (Figure 3). Regarding the precision protocol, only 10 articles reported the protocol used. (Supplementary Table 2) ${ }^{20}$ 


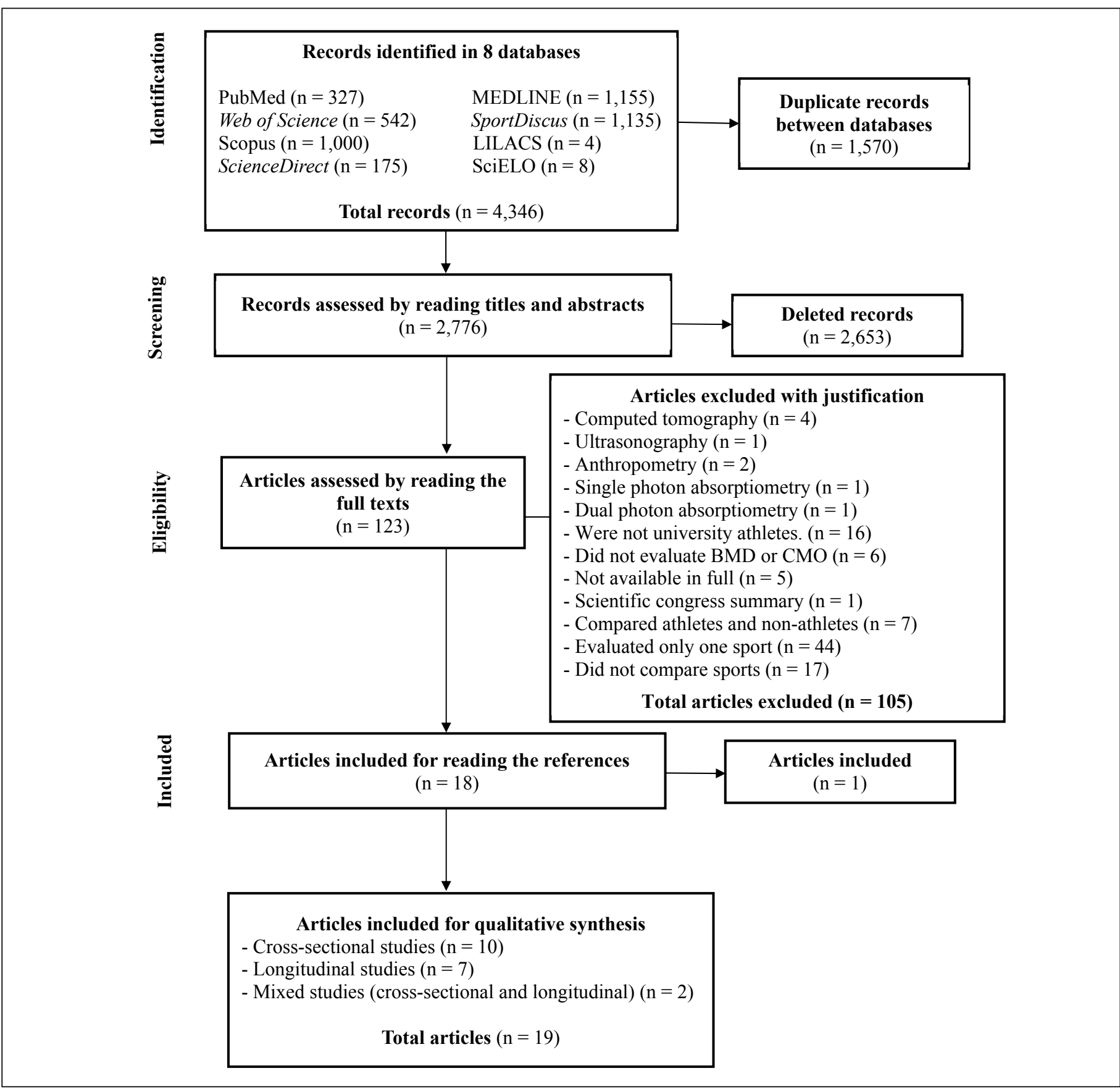

Figure 1. PRISMA flowchart diagram of records that investigated BMD and BMC in university athletes.

\section{Covariates tested}

The main covariates tested in the articles were total body mass (eight articles), lean mass (five articles) and height (four articles). The other covariates tested were summarized in Supplementary Table $3^{20}$. The covariates included in the adjusted models are described in Table 2.

\section{Comparison between sports}

Comparisons between sports were made by body region. Thus, of the 19 articles included in this review, 15 articles compared sports in the total body (BMD and BMC), nine articles in the upper limbs, 15 articles in the lower limbs (only BMD) and 15 articles in the trunk region (only BMD). (Table 2)

\section{Total Body}

In relation to $\mathrm{BMD}$, the most found result was that athletes of all sports (gymnastics, volleyball, judo, softball, athletics, field hockey and basketball) had a higher BMD of the total body when compared to swimming. ${ }^{23,27,33,34}$ Additionally, gymnastics athletes ${ }^{28,31,33}$ had a higher BMD of the total body than long distance runners. Also, football athletes with higher values than soccer and field hockey. ${ }^{40}$ Golf with lower DMO than other sports. ${ }^{40}$ (Table 2)

In relation to the $B M C$, the most found result was that athletes of basketball, softball, volleyball and athletics (sprinters and jumpers) presented higher values than swimming athletes. ${ }^{31,34}$ (Table 2)

\section{Umper Limbs}

In upper limbs, only BMD was investigated. In the arms, the most found result was that athletes of gymnastics, basketball, softball, volleyball and athletics (jumps and sprinters) had a higher BMD than swimming athletes. ${ }^{23,34,37,42}$ (Table 2)

In the forearms few studies have been found with this purpose. ${ }^{30,35}$ The swimmers had higher BMD values than long distance runners ${ }^{30}$ and the BMD was higher in wrestlers than in long distance runners and golf athletes. ${ }^{35}$ (Table 2) 
Table 1. Descriptive characteristics of systematic review studies.

\begin{tabular}{|c|c|c|c|c|c|c|c|}
\hline Authors & Place & Sex & Sports & Type of study & DXA Model & Software & $\begin{array}{c}\text { Bone } \\
\text { parameters }\end{array}$ \\
\hline $\begin{array}{l}\text { Fehling } \\
\text { et al. }{ }^{23}\end{array}$ & $\begin{array}{l}\text { United } \\
\text { States }\end{array}$ & $\mathrm{F}$ & $\begin{array}{l}\text { Volleyball }(n=8) \\
\text { Swimming }(n=7) \\
\text { Gymnastics }(n=13)\end{array}$ & Cross-sectional & $\begin{array}{l}\text { Hologic QDR 1000W (Waltham, } \\
\text { Massachusetts, United States) }\end{array}$ & $\begin{array}{c}\text { Version } 5.3 \\
\text { (whole body) Version } 4.26 \\
\text { (lumbar and femur) }\end{array}$ & BMD \\
\hline Lee et al. ${ }^{24}$ & $\begin{array}{l}\text { United } \\
\text { States }\end{array}$ & $\mathrm{F}$ & $\begin{array}{l}\text { Volleyball }(n=11) \\
\text { Basketball }(n=7) \\
\text { Soccer }(n=9) \\
\text { Swimming }(n=7) \\
\end{array}$ & Longitudinal & $\begin{array}{l}\text { Lunar DPX (Madison, } \\
\text { Wisconsin, United States) }\end{array}$ & Version Beta $3.0 \mathrm{H}$ & BMD \\
\hline Nichols et al. ${ }^{25}$ & $\begin{array}{l}\text { United } \\
\text { States }\end{array}$ & $\mathrm{F}$ & $\begin{array}{l}\text { Volleyball }(n=13) \\
\text { Basketball }(n=14) \\
\text { Gymnastics }(n=13) \\
\text { Tennis court }(n=6)\end{array}$ & Cross-sectional & $\begin{array}{l}\text { Lunar DPX (Madison, } \\
\text { Wisconsin, United States) }\end{array}$ & Version 3.4 & BMD \\
\hline Taaffe et al. ${ }^{26}$ & $\begin{array}{l}\text { United } \\
\text { States }\end{array}$ & $\mathrm{F}$ & $\begin{array}{l}\text { Gymnastics }(n=13) \\
\text { Swimming }(n=26)\end{array}$ & Cross-sectional & $\begin{array}{l}\text { Hologic QDR 1000W (Waltham, } \\
\text { Massachusetts, United States) }\end{array}$ & Version 5.47 & BMD \\
\hline $\begin{array}{l}\text { Matsumoto } \\
\text { et al. }{ }^{27}\end{array}$ & Japan & $M / F$ & $\begin{array}{l}\text { Athletics (long distance running) }(n=38 ; M=24, F=14) \\
\text { Judo }(n=37 ; M=14, F=23) \\
\text { Swimming }(n=28 ; M=21, F=7)\end{array}$ & Cross-sectional & $\begin{array}{l}\text { Norland XR26 (Fortatkinson, } \\
\text { Wisconsin, United States) }\end{array}$ & Unreported & BMD \\
\hline Taaffe et al. ${ }^{28}$ & $\begin{array}{l}\text { United } \\
\text { States }\end{array}$ & $\mathrm{F}$ & $\begin{array}{c}\text { Cohort } 1 \\
\text { - Gymnastics }(n=26) \\
\text { - Athletics (running - } 800 \text { m) }(n=36) \\
\text { Cohort } 2 \\
\text { - Gymnastics }(n=8) \\
\text { - Swimming }(n=11) \\
\end{array}$ & Longitudinal & $\begin{array}{l}\text { Hologic QDR 1000W (Waltham, } \\
\text { Massachusetts, United States) }\end{array}$ & Unreported & BMD \\
\hline $\begin{array}{c}\text { Emslander } \\
\text { et al. }{ }^{29} \\
\end{array}$ & $\begin{array}{l}\text { United } \\
\text { States }\end{array}$ & $\mathrm{F}$ & $\begin{array}{l}\text { Athletics (long distance running) }(n=21) \\
\text { Swimming }(n=22)\end{array}$ & Cross-sectional & $\begin{array}{l}\text { Hologic QDR 1000W (Waltham, } \\
\text { Massachusetts, United States) }\end{array}$ & Unreported & BMD \\
\hline Rourke et al. ${ }^{30}$ & $\begin{array}{l}\text { United } \\
\text { States }\end{array}$ & F & $\begin{array}{l}\text { Athletics (long distance running) }(n=16) \\
\text { Swimming }(n=14)\end{array}$ & Longitudinal & $\begin{array}{l}\text { Hologic QDR 1000W (Waltham, } \\
\text { Massachusetts, United States) }\end{array}$ & Unreported & BMD \\
\hline $\begin{array}{l}\text { Bemben } \\
\text { et al. }^{31}\end{array}$ & $\begin{array}{l}\text { United } \\
\text { States }\end{array}$ & $\mathrm{F}$ & $\begin{array}{l}\text { Athletics (long distance running) }(n=15) \\
\text { Gymnastics }(n=11)\end{array}$ & Longitudinal & $\begin{array}{c}\text { Lunar DPX-IQ (Madison, } \\
\text { Wisconsin, United States) }\end{array}$ & Version 4.1 & BMD \\
\hline $\begin{array}{l}\text { Beals and } \\
\text { Hill }^{32}\end{array}$ & $\begin{array}{l}\text { United } \\
\text { States }\end{array}$ & $\mathrm{F}$ & $\begin{array}{l}\text { Lean-build sports ( } \mathrm{n}=65 \text { ) } \\
\text { - diving, atletismo, athletics (long distance } \\
\text { running), swimming, athletics (sprinters) } \\
\text { Non-lean buildsports ( } \mathrm{n}=47 \text { ) } \\
\text { - (field hockey, softball, tennis court } \\
\text { e athletics (field categories) } \\
\end{array}$ & Cross-sectional & $\begin{array}{l}\text { Lunar DPX (Lunar-GE Corp., } \\
\text { Madison, Wisconsin, United } \\
\text { States) }\end{array}$ & Unreported & BMD \\
\hline Mudd et al. ${ }^{33}$ & $\begin{array}{l}\text { United } \\
\text { States }\end{array}$ & $\mathrm{F}$ & $\begin{array}{c}\text { Gymnastics }(n=8) \\
\text { Softball }(n=14) \\
\text { Athletics (long distance running }>800 \text { meters) }(n=25) \\
\text { Athletics (speed runners }<800 \text { meters) }(n=8) \\
\text { Field Hockey }(n=10) \\
\text { Soccer }(n=10) \\
\text { Rowing }(n=15) \\
\text { Swimming/diving }(n=9) \\
\end{array}$ & Cross-sectional & $\begin{array}{l}\text { Hologic QDR-1000W (Bedford, } \\
\text { Massachusetts, United States) }\end{array}$ & Version 6.0 & BMD \\
\hline $\begin{array}{l}\text { Carbuhn } \\
\text { et al. }{ }^{34}\end{array}$ & $\begin{array}{l}\text { United } \\
\text { States }\end{array}$ & F & $\begin{array}{r}\text { Softball }(n=17) \\
\text { Basketball }(n=10) \\
\text { Volleyball }(n=10) \\
\text { Swimming }(n=16) \\
\text { Athletics (jumps and sprinters) }(n=17)\end{array}$ & Longitudinal & $\begin{array}{l}\text { Lunar Prodigy Advance } \\
\text { (GE Healthcare, Madison, } \\
\text { Wisconsin, United States) }\end{array}$ & Unreported & $B M D$ and $B M C$ \\
\hline $\begin{array}{l}\text { Ackerman } \\
\text { et al. }{ }^{35}\end{array}$ & $\begin{array}{l}\text { United } \\
\text { States }\end{array}$ & M & $\begin{array}{c}\text { Athletics (long distance runners) }(n=13) \\
\text { Wrestlers }(n=6) \\
\text { Golf }(n=7)\end{array}$ & Cross-sectional & $\begin{array}{l}\text { Hologic QDR 1000W (Waltham, } \\
\text { Massachusetts, United States) }\end{array}$ & Unreported & BMD \\
\hline Hirsch et al. ${ }^{36}$ & $\begin{array}{l}\text { United } \\
\text { States }\end{array}$ & $M / F$ & $\begin{array}{c}\text { Sprinters (sprint/sprint hurdles) }(n=17) \\
\text { Mid-distance runners (middistance/hurdles) }(n=9) \\
\text { Multis (heptathlon and decathlon) }(n=9) \\
\text { Jumpers (jumps/high jump) }(n=7) \\
\text { Pole vault/javelin }(n=9) \\
\text { Throws }(n=9)\end{array}$ & $\begin{array}{c}\text { Cross-sectional } \\
\text { and longitudinal }\end{array}$ & $\begin{array}{l}\text { Hologic Discovery W (Bedford, } \\
\text { Massachusetts, United States) }\end{array}$ & $\begin{array}{l}\text { APEX Biologix } \\
\text { version } 3.3\end{array}$ & BMC \\
\hline $\begin{array}{l}\text { Stanforth } \\
\text { et al. }{ }^{37}\end{array}$ & $\begin{array}{l}\text { United } \\
\text { States }\end{array}$ & F & $\begin{array}{c}\text { Basketball }(n=38) \\
\text { Soccer }(n=47) \\
\text { Swimming }(n=52) \\
\text { Athletics (sprinters and jumpers) }(n=49) \\
\text { Volleyball }(n=26)\end{array}$ & $\begin{array}{c}\text { Cross-sectional } \\
\text { and longitudinal }\end{array}$ & $\begin{array}{l}\text { Lunar Prodigy Pro (GE- Healthcare, } \\
\text { Madison, Wisconsin, United States) }\end{array}$ & enCORE version 11.0 & BMD and BMC \\
\hline $\begin{array}{c}\text { Nepocatych } \\
\text { et al. }{ }^{38} \\
\end{array}$ & $\begin{array}{l}\text { United } \\
\text { States }\end{array}$ & $\mathrm{F}$ & $\begin{array}{c}\text { Basketball }(n=10) \\
\text { Softball }(n=10)\end{array}$ & Longitudinal & \begin{tabular}{|c|} 
Lunar Prodigy Pro (GE Healthcare, \\
Madison, Wisconsin, United States) \\
\end{tabular} & Unreported & BMD \\
\hline $\begin{array}{l}\text { Scerpella } \\
\text { et al. }{ }^{39}\end{array}$ & $\begin{array}{l}\text { United } \\
\text { States }\end{array}$ & $M / F$ & $\begin{array}{c}\text { Basketball }(n=22) \\
\text { Field Hockey }(n=45) \\
\text { Soccer }(n=18) \\
\end{array}$ & Longitudinal & $\begin{array}{c}\text { Lunar Prodigy Pro (GE Healthcare, } \\
\text { Madison, Wisconsin, United States) }\end{array}$ & Version 13.2-14.1 & BMD \\
\hline $\begin{array}{l}\text { Sanfilippo } \\
\text { et al. } 40\end{array}$ & $\begin{array}{l}\text { United } \\
\text { States }\end{array}$ & $M / F$ & $\begin{array}{l}\text { Football }(n=117) \\
\text { Wrestling }(n=31) \\
\text { Soccer }(n=56) \\
\text { Field Hockey }(n=48) \\
\text { Basketball }(n=30) \\
\text { Golf }(n=19) \\
\text { Softball }(n=20) \\
\text { Volleyball }(n=16) \\
\end{array}$ & Cross-sectional & $\begin{array}{c}\text { Lunar Prodigy Pro (GE Healthcare, } \\
\text { Madison, Wisconsin, United States) }\end{array}$ & Version 14.1 & $B M C$ and $B M D$ \\
\hline Fields et al. ${ }^{41}$ & $\begin{array}{l}\text { United } \\
\text { States }\end{array}$ & $\mathrm{F}$ & $\begin{array}{l}\text { Basketball }(n=12) \\
\text { Athletics }(n=12) \\
\text { Volleyball }(n=12)\end{array}$ & Cross-sectional & $\begin{array}{l}\text { Hologic QDR 1000W (Waltham, } \\
\text { Massachusetts, United States) }\end{array}$ & & BMD \\
\hline
\end{tabular}

F-female; M - male; $\mathrm{n}$ - sample number; BMC - bone mineral content; BMD - bone mineral density. 
Table 2. Synthesis of the comparison of BMD and BMC between sports by body region and covariates of the final model.

\begin{tabular}{|c|c|c|c|}
\hline \multicolumn{4}{|c|}{ Results } \\
\hline Authors & BMD & BMC & Covariates \\
\hline \multicolumn{4}{|c|}{ Total body } \\
\hline Fehling et al. ${ }^{23}$ & Volleyball and Gymnasstic > Swimming & NA & $\begin{array}{l}\text { Body mass } \\
\text { and height }\end{array}$ \\
\hline Lee et al. ${ }^{24}$ & No differences between sports & NA & $\begin{array}{l}\text { Body mass } \\
\text { and height }\end{array}$ \\
\hline Nichols et al. ${ }^{25}$ & No differences between sports & NA & Body mass \\
\hline Taaffe et al. ${ }^{26}$ & No differences between sports & $\mathrm{NA}$ & None \\
\hline Taaffe et al. ${ }^{28}$ & $\begin{array}{l}\text { Cohort 1: Gymnastic > Long distance running } \\
\text { Cohort 2: No differences between sports }\end{array}$ & NA & None \\
\hline $\begin{array}{l}\text { Matsumoto } \\
\text { et al. }{ }^{27}\end{array}$ & Judo > Long distance running and Swimming & NA & None \\
\hline $\begin{array}{l}\text { Emslander } \\
\text { et al. }{ }^{29}\end{array}$ & No differences between sports & NA & None \\
\hline Bemben et al. ${ }^{31}$ & Gymnasstic > Long distance running & $\mathrm{NA}$ & Height \\
\hline Mudd et al. ${ }^{33}$ & $\begin{array}{c}\text { Gymnasstic }>\text { Long distance running } \\
\text { Gymnasstic, Softball, Athletics (sprinters) and } \\
\text { Field Hockey }>\text { Swimming/Diving }\end{array}$ & NA & $\begin{array}{l}\text { Body mass and } \\
\text { menstrual status }\end{array}$ \\
\hline Carbuhn et al. ${ }^{34}$ & Basketball, Softball, Volleyball and Athletics (jumps and sprinters) > Swimming & Basketball, Softball, Volleyball and Athletics (jumps and sprinters) > Swimming & None \\
\hline Hirsch et al. ${ }^{36}$ & NA & $\begin{array}{l}\text { Sprints/Hurdles, Jumps /High jump, Pole vault/Javelin > Throws } \\
\text { Sprints/Hurdles, Pole vault/Javelin }>\text { Heptathletes/Decathletes }\end{array}$ & None \\
\hline Stanforth et al. ${ }^{37}$ & No differences between sports & Basketball > Swimming, Soccer, Volleyball and Athletics & $\begin{array}{l}\text { Body mass } \\
\text { and race }\end{array}$ \\
\hline $\begin{array}{l}\text { Nepocatych } \\
\text { et al. }{ }^{38}\end{array}$ & No differences between sports & NA & None \\
\hline Sanfilippo et al. ${ }^{40}$ & $\begin{array}{c}\text { Football > Soccer and Field Hockey } \\
\text { Footbal, Wrestling, Soccer, Hockey, Basketball, Softball, Volleyball, } \\
\text { Basketball, Track and field and Volleyball > Golf }\end{array}$ & No differences between sports & None \\
\hline Fields et al. ${ }^{41}$ & No differences between sports & NA & None \\
\hline \multicolumn{4}{|c|}{ Umper limbs } \\
\hline fehling et al. ${ }^{23}$ & Arms: Gymnastic > Volleyball and Swimming & NA & $\begin{array}{l}\text { Body mass } \\
\text { and height }\end{array}$ \\
\hline Lee et al. ${ }^{24}$ & Arms: No differences between sports & NA & $\begin{array}{l}\text { Body mass } \\
\text { and height }\end{array}$ \\
\hline Nichols et al. ${ }^{25}$ & Arms: No differences between sports & NA & Body mass \\
\hline Taaffe et al. ${ }^{26}$ & Arms: Gymnastic > Swimming: & NA & None \\
\hline Rourke et al. ${ }^{30}$ & Forearm (radial): Swimming $>$ Runners & NA & Body mass \\
\hline Carbuhn et al. ${ }^{34}$ & $\begin{array}{l}\text { Arms: Basketball, Softball, Volleyball and Athletics } \\
\text { (jumps and sprinters) > Swimming }\end{array}$ & NA & None \\
\hline $\begin{array}{l}\text { Ackerman } \\
\text { et al. }{ }^{35}\end{array}$ & Forearm (radial): Wrestlers $>$ Runners and Golf & NA & None \\
\hline Stanforth et al. ${ }^{37}$ & Arms: Basketball > Swimming, Soccer, Volleyball and Athletics & NA & $\begin{array}{l}\text { Body mass } \\
\text { and race }\end{array}$ \\
\hline \multicolumn{4}{|c|}{ Lower limbs } \\
\hline fehling et al. ${ }^{23}$ & $\begin{array}{l}\text { Legs: Volleyball and Gymnastic > Swimming } \\
\text { Pelvis: Volleyball and Gymnastic > Swimming }\end{array}$ & NA & $\begin{array}{l}\text { Body mass } \\
\text { and height }\end{array}$ \\
\hline Lee et al. ${ }^{24}$ & $\begin{array}{c}\text { Legs: No differences between sports } \\
\text { Femur neck: No differences between sports } \\
\text { Femur trochanter: No differences between sports } \\
\text { Femur Ward's: Basketball > Volleyball, Soccer and Swimming } \\
\text { Pelvis: No differences between sports }\end{array}$ & NA & $\begin{array}{l}\text { Body mass } \\
\text { and height }\end{array}$ \\
\hline Nichols et al. ${ }^{25}$ & $\begin{array}{l}\text { Legs: No differences between sports } \\
\text { Femur neck: No differences between sports }\end{array}$ & NA & Body mass \\
\hline Taaffe et al. ${ }^{26}$ & $\begin{array}{l}\text { Legs: Gymnastic > Swimming } \\
\text { Femur neck: Gymnastic > Swimming } \\
\text { Femur trochanter: Gymnastic }>\text { Swimming }\end{array}$ & NA & None \\
\hline Taaffe et al. ${ }^{28}$ & $\begin{array}{l}\text { Femur neck: Cohort 1: Gymnastic > Runners } \\
\text { Cohort 2: Gymnastic > Swimming }\end{array}$ & & None \\
\hline $\begin{array}{c}\text { Emslander } \\
\text { et al. }{ }^{29}\end{array}$ & Total proximal femur: No differences between sports & & None \\
\hline Rourke et al. ${ }^{30}$ & $\begin{array}{l}\text { Total proximal femur: No differences between sports } \\
\text { Femur neck: No differences between sports } \\
\text { Femur trochanter: No differences between sports } \\
\text { Femur Ward's: No differences between sports }\end{array}$ & NA & Body mass \\
\hline Bemben et al. ${ }^{31}$ & $\begin{array}{l}\text { Femur neck: Gymnasstic }>\text { Runners } \\
\text { Total proximal femur: Gymnastic }>\text { Runners } \\
\text { Femur trochanter: Gymnastic }>\text { Runners } \\
\text { Femur ward's: Gymnastic }>\text { Runners }\end{array}$ & NA & Height \\
\hline Mudd et al. ${ }^{33}$ & $\begin{array}{l}\text { Pelvis: Gymnastic, Softball and Field Hockey > Swimming/Diving } \\
\text { Gymnastic, Softball, Field Hockey and Soccer }>\text { Runners }\end{array}$ & NA & $\begin{array}{l}\text { Body mass } \\
\text { and Menstrual } \\
\text { status* } \\
\end{array}$ \\
\hline Carbuhn et al. ${ }^{34}$ & $\begin{array}{c}\begin{array}{c}\text { Legs: Basketball, Softball, Volleyball and Athletics } \\
\text { (jumps and sprinters) }>\text { Swimming }\end{array} \\
\text { Pelvis: Basketball, Softball, Volleyball and Track and field > Swimming }\end{array}$ & NA & None \\
\hline
\end{tabular}




\begin{tabular}{|c|c|c|c|}
\hline $\begin{array}{l}\text { Ackerman } \\
\text { et al. }{ }^{35}\end{array}$ & $\begin{array}{l}\text { Total proximal femur: Wrestlers > Runners and Golf } \\
\text { Femur neck: Wrestlers > Runners and Golf }\end{array}$ & NA & None \\
\hline Stanforth et al. ${ }^{37}$ & $\begin{array}{l}\text { Legs: Basketball > Swimming, Soccer, Volleyball and Athletics } \\
\text { Pelvis: Basketball > Swimming, Soccer, Volleyball and Athletics }\end{array}$ & NA & $\begin{array}{l}\text { Body mass } \\
\text { and race }\end{array}$ \\
\hline $\begin{array}{l}\text { Nepocatych } \\
\text { et al. }{ }^{38}\end{array}$ & Total proximal femur: No differences between sports & NA & None \\
\hline Scerpella et al. ${ }^{39}$ & Legs: Soccer > Basketball and Field Hockey & NA & $\begin{array}{l}\text { Age, gender, } \\
\text { race and sport }\end{array}$ \\
\hline Fields et al. ${ }^{41}$ & Hip: No differences between sports & NA & None \\
\hline \multicolumn{4}{|c|}{ Trunk } \\
\hline Fehling et al. ${ }^{23}$ & Torso: No differences between sports & NA & $\begin{array}{l}\text { Body mass } \\
\text { and height }\end{array}$ \\
\hline Lee et al. ${ }^{24}$ & Lumbar Spine (L2-L4): No differences between sports & NA & $\begin{array}{l}\text { Body mass } \\
\text { and height }\end{array}$ \\
\hline Nichols et al. ${ }^{25}$ & Lumbar Spine (L2-L4): No differences between sports & NA & Body mass \\
\hline Taaffe et al. ${ }^{26}$ & $\begin{array}{l}\text { Lumbar spine (L2-L4): No differences between sports } \\
\text { Spine }{ }^{* *} \text { Gymnastic > Swimming } \\
\text { Thoracic Spine }{ }^{* *} \text { : No differences between sport } \\
\text { Ribs: No differences between sports }\end{array}$ & NA & None \\
\hline Taaffe et al. ${ }^{28}$ & $\begin{array}{c}\text { Lumbar spine (L2-L4): } \\
\text { Cohort 1: Gymnastic }>\text { Runners } \\
\text { Cohort 2: No differences between sports }\end{array}$ & NA & None \\
\hline $\begin{array}{c}\text { Emslander } \\
\text { et al. }{ }^{29}\end{array}$ & Lumbar spine (L2-L4): No differences between sports & NA & None \\
\hline Rourke et al. ${ }^{30}$ & Lumbar spine (L1-L4): No differences between sports & NA & Body mass \\
\hline Bemben et al. ${ }^{31}$ & Lumbar spine (L2-L4): Gymnasstic > Runners & NA & Height \\
\hline Beals and Hill ${ }^{32}$ & $\begin{array}{c}\text { Lumbar spine (L1-L4): Field Hockey, Softball, Tennis, athletics (field events) } \\
\text { > Diving, Cross-country, Swimming, athletics (sprinting events) }\end{array}$ & NA & Lean mass \\
\hline $\begin{array}{l}\text { Mudd et } \\
\text { al.2007 } 33\end{array}$ & $\begin{array}{l}\text { Lumbar spine**: Gymnastic }>\text { Swimming/Diving } \\
\text { Gymnastic and Softball > Runners }\end{array}$ & NA & $\begin{array}{l}\text { Menstrual } \\
\text { status* and } \\
\text { body mass }\end{array}$ \\
\hline Carbuhn et al. ${ }^{34}$ & Spine ${ }^{* *}$ Basketball, Softball, Volleyball and Track and field > Swimming & NA & None \\
\hline $\begin{array}{l}\text { Ackerman } \\
\text { et al. }{ }^{35}\end{array}$ & Spine ${ }^{* *}$ Wrestlers $>$ Runners and Golf & NA & None \\
\hline Stanforth et al. ${ }^{37}$ & $\begin{array}{c}\text { Spine }{ }^{* *} \text { : No differences between sports } \\
\text { Torso: Basketball > Swimming, Soccer, Volleyball and Athletics }\end{array}$ & NA & $\begin{array}{l}\text { Body mass } \\
\text { and race }\end{array}$ \\
\hline $\begin{array}{c}\text { Nepocatych } \\
\text { et al. }{ }^{38}\end{array}$ & Lumbar spine (L1-L4): No differences between sports & NA & None \\
\hline Fields et al. ${ }^{41}$ & Lumbar spine**: No differences between sports & $\mathrm{NA}$ & None \\
\hline
\end{tabular}

NA - not applicable; >: bigger then; L: lumbar vertebra; ${ }^{-}$- amenorrhea (0 to 3 cycles per year), oligomenorrhea (4 to 9 cycles per year), eumenorrhea (10 to 12 cycles per year); ** results acquired through the whole body scan.

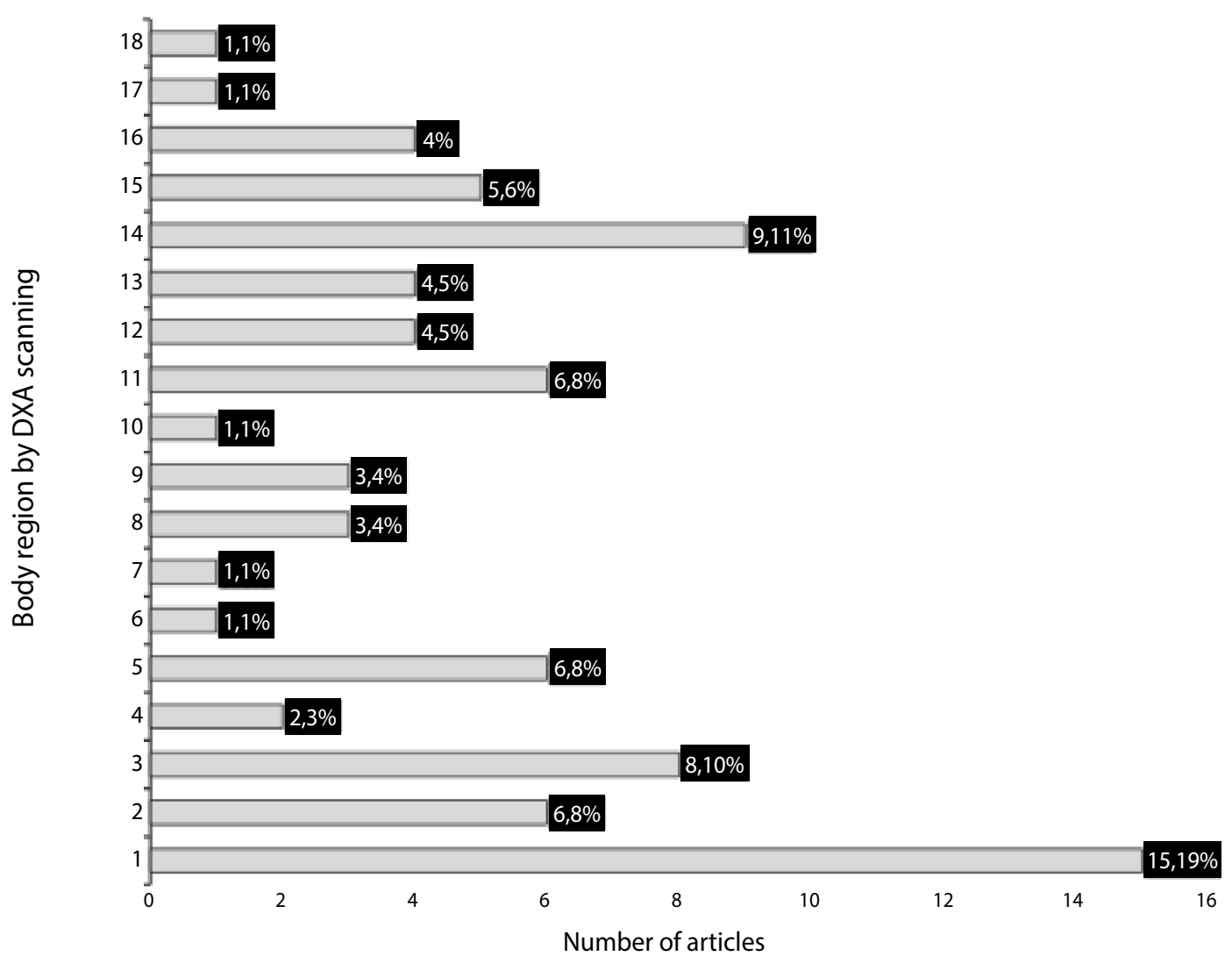

Figure 2. Body regions analyzed by DXA stratified by scanning. 


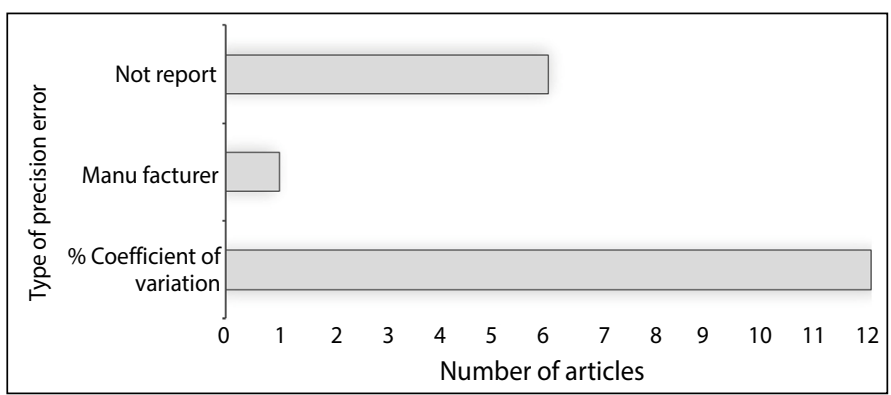

Figure 3. Type of precision error.

\section{Lower limbs}

In lower limbs, only BMD was investigated. The articles included in the present review compared BMD in seven specific regions of the lower limbs (legs, femur neck, greater trochanter, Ward's triangle, total proximal femur, pelvis and hips). (Table 2)

In the legs, the highlights were that the BMD of athletes who practiced volleyball, gymnastics, basketball, softball and athletics (jumpers and sprinters) was higher when compared to swimming athletes. ${ }^{23,26,34}$ (Table 2)

In the femur neck, the most found result was that gymnastics athletes ${ }^{28,31}$ had a higher BMD than long distance running athletes. Gymnastics had a higher BMD than swimming. ${ }^{26,28}$ (Table 2)

For the regions of greater trochanter, ${ }^{26,31}$ Ward's triangle, ${ }^{24,31}$ and proximal femur total ${ }^{11,35} \mathrm{few}$ studies have been found with this purpose. The result most found in these few studies was that gymnastics and athletes had higher BMD values in these body regions than athletes of other sports (swimming, long distance running). (Table 2)

In pelvis region, athletes of volleyball, gymnastic, softball, field hockey, basketball and athletics had a higher BMD than swimming athletes. ${ }^{23,33,34}$ In addition, athletes of gymnastic, softball, field hockey and soccer showed higher BMD than long distance runners. ${ }^{33}$ Also, athletes of basketball showed a higher BMD than athletes of football, volleyball and athletics. ${ }^{36}$ (Table 2)

\section{Trunk}

In the trunk, only BMD was investigated. The articles included in the present review compared BMD in five specific regions of the trunk (torso, ribs, lumbar spine, thoracic spine and total spine). For the torso region, only one study found that athletes of basketball showed higher BMD values than athletes of swimming, soccer, volleyball and athletic. ${ }^{37}$ For the lumbar spine region, the most found result was that athletes of gymnastics, softball, field hockey, tennis and athletics (sprinting events) have a higher BMD than swimming, 32,33 and athletes of gymnastics showed higher BMD values than long distance runners. ${ }^{28,31,33}$ In the total spine (by whole body scan), athletes of gymnastics, basketball, softball, volleyball and track and field had higher BMD values than swimming athletes. ${ }^{26,34}$ There were no differences between sports when comparing the BMD of the ribs and thoracic spine. (Table 2)

\section{Risk of bias}

When considering the general classification of all types of included studies (19 articles), it was verified that two studies ${ }^{31,39}$ (longitudinal) presented low risk of bias, 16 studies $23,24,26,28-30,32-38,40,41$ presented moderate risk of bias (nine cross-sectional, five longitudinal and two mixed studies) and one study ${ }^{27}$ (cross-sectional) presented high risk of bias. (Supplementary Table 4) ${ }^{20}$

\section{DISCUSSION}

The main results of this review were: 1) BMD as the most investigated bone parameter; 2 ) total body, anteroposterior lumbar spine ( $L 1$ to $L 4$ or
L2 to L4) and proximal femur (mainly femoral neck) as the most studied body regions; 3 ) coefficient of variation as the main indicator of precision error; 4) total body mass and height as the covariates most used in the final statistical models; 5) swimming and long distance running showed lower BMD and BMC values than athletes from the other investigated sports, regardless of the body region analyzed.

The BMD and BMC were the only parameters reported in the studies of this review In fact, BMD and BMC are the bone parameters most commonly used in athletes, since they express weight and density of bone minerals. ${ }^{43}$ However, other bone parameters related to bone stiffness and strength (bone geometry and hip strength) that are also evaluated by DXA ${ }^{44}$ and may also explain the osteogenic differences between sports types. ${ }^{45,46}$ Investigating other bone parameters in athletes may bring new insights into the bone quality of this group.

The present review revealed that the body regions most investigated in university athletes were BMD and BMC of the total body and BMD of the anteroposterior lumbar spine and proximal femur. The ISCD recommends that examinations for these three body regions should be performed. In the whole body examination, the results of total body and subregions such as arms, legs and trunk can be used in order to compare the impact of the sport. In addition, the anteroposterior lumbar spine and the total proximal femur are regions that are more sensitive to the effects of regular practice of sports because the vertebrae and the femoral neck region have higher concentrations of trabecular bone, which is responsible for providing modifications by metabolic changes in the osteogenesis process. ${ }^{47}$ In addition, it is necessary to investigate $B M D$ and $B M C$ of these body regions in a serial way to quantify the annual effects of training, detraining and physical maturation on the bone composition of athletes ${ }^{47}$ For this reason, studies with university athletes should go beyond total body BMD and/or BMC estimates.

Regarding the precision error, the main results were that 12 articles used \% CV and six articles did not report the precision error. The ISCD recommends that the precision error value be expressed as root mean squared value with standard deviation (RMS $\pm S D$ ) in the same unit of measure of BMD $\left(\mathrm{g} / \mathrm{cm}^{2}\right) .17,48$ However, sometimes as in the case of articles inserted in the present review (approximately 63\%), the error is expressed as $\mathrm{CV}$ or $\% \mathrm{CV}$, but this is less desirable due to the variation of these values over a range of BMD measurements, which generates greater precision error. ${ }^{15,17}$ In addition, the high number of articles (approximately $32 \%$ ) that did not report an accuracy error is a weakness identified in the articles included in this review, as the conclusions identified in the studies may be variations due to operator or instrument error. ${ }^{1749,50}$ Therefore, it is important that serial measurements be performed at the BMD to calculate the LSC and ensure the accuracy of the results.

The articles included of this review showed heterogeneity regarding the protocols used to calculate the precision error, and no study met the recommendations standardized by ISCD. ${ }^{16,51}$ A possible justification, is that the ISCD recommendation was published in 2013, 16,48,51 however, most of the articles (13 articles) were published until 2012. Additionally, of the six articles published since 2013, it was identified that five articles did not report the protocol used and one article did not follow the recommendations. Therefore, when studying athletes it is important to follow the ISCD recommendations for precision protocols, in order to make the comparison of bone parameters between athletes more accurate, reliable and free of errors that could be controlled..$^{16,51}$

Total body mass and height were the most used covariates in the final statistical models. Total body mass is directly associated with the parameters of BMD and BMC. ${ }^{9}$ However, caution is necessary, because when assessing this covariate alone, it is not possible to identify and quantify the distribution of other components of body composition that 
may interfere with the process of bone formation, such as fat mass and lean mass. ${ }^{8,52,53}$ In athletes, the increase of lean body mass in relation to fat body mass provides greater mechanical load, thus stimulating bone formation. 8 .9 Fat body mass increases serum leptin levels and regulates the development of osteoclasts (remodeling and bone absorption cells), contributing to bone formation. ${ }^{9}$ It is important to emphasize that the contribution of fat body mass is less important than that of lean body mass, since it is often associated with other metabolic disorders, such as diabetes, cardiovascular diseases and some neoplasms. ${ }^{8,9}$ Therefore, these variables can interfere in the interpretation of the impact of university sports on bone parameters.

In addition to these variables, height is a variable that directly influences BMC; therefore, taller athletes may erroneously present greater BMC when compared to smaller athletes, so BMC should be corrected by athletes' height. ${ }^{54}$ This cannot be applied to BMD as it already has bone mass values corrected by the athlete's square area. Thus, considering the limitations of body mass and height, future investigations need to include in statistical models, covariates that exert greater interference in bone parameters in athletes (e.g. fat and lean mass, time of practice, training volume, menstrual state and use of supplementation and drugs). 8,9 Furthermore, most of the studies included in this review were classified as moderate and the item that the articles most need to improve is the control of possible confounding variables.

Regardless of the body region analyzed, most studies reported that swimming and long-distance running athletes had lower BMD and $B M C$ values when compared to other sports. Systematic review studies with meta-analysis also identified swimming with lower BMD values for the total body, femoral neck and lumbar spine compared to sports with greater osteogenic potential. ${ }^{3}$ This is due to the high exposure of swimmers to hypogravity and the lack of impact, characteristics inherent to their training sessions. ${ }^{4}$ The consequence of this is that swimmers have a higher risk of fractures during athletic life and greater chances of osteoporosis in post-athletic life compared to athletes in other sports. ${ }^{3}$ Therefore, it is recommended that swimmers include weight-bearing exercises in their training routine., ${ }^{3,4}$

In relation to long distance runners, the lower BMD and BMC values compared to other sports can be explained by the difference in load magnitude, speed and frequency between sports ${ }^{11,45}$ Training sessions for sports such as gymnastics, basketball and volleyball include activities with greater bone deformation caused by the effect of axial loads on the bone and, consequently, increase the osteogenic potential of these sports. ${ }^{11}$ Long distance running is characterized by a large number of consecutive impacts of low to moderate magnitude and is therefore less osteogenic. ${ }^{13}$ For this reason, studies have classified the osteogenic potential of long running as superior only to swimming. 11,13 However, professional long distance running athletes are at increased risk for developing knee and hip osteoarthritis.?
Something to note is that, although studies have not identified differences in the BMD of swimmers ${ }^{3,4}$ and runners ${ }^{7}$ when compared to sedentary controls, bone remodeling is greater in swimmers and runners than non-athletes, which can result in a stronger structure and, consequently, a stronger bone. In other words, playing sports remains better for your health than not playing.

Some highlights were identified in the comparison of sports with emphasis on the same body region. Basketball athletes had higher BMD values for arms, legs, Ward's triangle and torso than volleyball players. ${ }^{24,37}$ Still, the BMD of the arms was higher in gymnasts than in volleyball athletes. ${ }^{23}$ Through these results it is speculated that even if similar, the osteogenic effect of basketball in the upper limbs, lower limbs and torso to be greater than in volleyball athletes. Also, it is speculated greater bone benefit in the upper limbs in gymnasts when compared to volleyball athletes.

The heterogeneity of the studies is considered a study limitation, since they can make comparisons and interpretations of the results related to BMD and BMC among athletes difficult. However, regardless of the type of study, the articles in this review responded to the proposed objectives. The investigation of the precision error and the protocol used to evaluate DXA in university athletes is another strong point of this systematic review. The comparison between sports by body region is a novelty of the study. In addition, the description of the covariates used in the final model, registration in PROSPERO, the large number of databases included and the analysis of the risk of bias are other strengths of this work.

In conclusion, BMD was the most investigated bone parameter, and the total body, lumbar spine and total proximal femur were the most investigated body regions among university athletes. In addition, although new reflections were given, \%CV was the most widely used precision error measure, and body mass and height were the most covariates cited in the final statistical models. Finally, long distance running and swimming athletes had lower BMD and BMC than athletes from other sports. It is speculated, greater osteogenic potential of basketball and gymnastics when compared to volleyball.

Thus, coaches, sports medicine professionals, sports nutritionists and other health professionals can use the results of this review as intervention strategies to control training and covariates that can influence the relationship between bone health and sport, which it can prevent diseases and health problems during and after athletic life. In addition, this review provides theoretical support for the technical control of DXA measurement in athletes.

\section{Funding}

This research was financed in part by the Coordenação de Aperfeiçoamento de Pessoal de Nível Superior__Brazil (CAPES)_Finance Code 001.

$\overline{\text { All authors declare no potential conflict of interest related to this article }}$

\section{REFERENCES}

1. Schtscherbyna A, Ribeiro BG, Fleiuss FML. Bone Health, Bone Mineral Density, and Sports Performance. Nutrition and Enhanced Sports Performance, Elsevier. 2019:73-81.

2. Agostinete RR, Maillane-Vanegas S, Lynch KR, Turi-Lynch B, Coelho-E-Silva MJ, Campos EZ, et al. The Impact of Training Load on Bone Mineral Density of Adolescent Swimmers: A Structural Equation Modeling Approach. Pediatr Exerc Sci. 2017;29(4):520-8.

3. Gomez-Bruton A, Montero-Marin J, Gonzalez-Agueero A, Gómez-Cabello A, Garcia-Campayo A, Moreno $L A$, et al. Swimming and peak bone mineral density: a systematic review and meta-analysis. J Sports Sci. 2018;36(4):365-77.

4. Gómez-Bruton A, Gónzalez-Agüero A, Gómez-Cabello A, Casajús JA, Vicente-Rodríguez G. Is Bone Tissue Really Affected by Swimming? A Systematic Review. PLoS ONE. 2013;8(8):1-25.
5. Vlachopoulos D, Barker AR, Ubago-Guisado E, Fatouros IG, Knapp KM, Williams CA, et al. Longitudinal Adaptations of Bone Mass, Geometry, and Metabolism in Adolescent Male Athletes: The PRO-BONE Study. J Bone Miner Res. 2017;32(11):2269-77.

6. Zouch M, Chaari H, Zribi A, Bouajina E, Vico L, Alexandre C, et al. Volleyball and Basketball Enhanced Bone Mass in Prepubescent Boys. J Clin Densitom. 2016;19(3):396-403.

7. Alentorn-Geli E, Samuelsson K, MusahI V, Green CL, Bhandari M, Karlsson J. The Association of Recreational and Competitive Running With Hip and Knee Osteoarthritis: A Systematic Review and Meta-analysis. J Orthop Sports Phys Ther. 2017;47(6):373-90.

8. Goolsby MA, Boniquit N. Bone health in athletes: the role of exercise, nutrition, and hormones. Sports Health. 2017;9(2):108-17. 
9. Sarkis KS, de Medeiros Pinheiro M, Szejnfeld VL, Martini LA. High bone density and bone health. Endocrinol Nutr. 2012;59(3):207-14.

10. American Bone Health. Glossary - American Bone Health; 2018 [Accessed June 16, 2019]. Avaiable in: https://americanbonehealth.org/glossary/

11. Dolan SH, Williams DP, Ainsworth BE, Shaw JM. Development and reproducibility of the bone loading history questionnaire. Med Sci Sports Exerc. 2006;38(6):1121-31.

12. Nikander R, Sievänen $H$, Heinonen A, Kannus P. Femoral neck structure in adult female athletes subjected to different loading modalities. J Bone Miner Res. 2005;20(3):520-8.

13. Nikander R, Kannus P, Rantalainen T, Uusi-Rasi K, Heinonen A, Sievänen H. Cross-sectional geometry of weight-bearing tibia in female athletes subjected to different exercise loadings. Osteoporos Int. 2010;21(10):1687-94.

14. Williams CAP, Vlachopoulos D, Barker AR, Knapp KM, Metcalf BS, Fatouros IG, et al. The impact of sport participation on bone mass and geometry in adolescent males. Med Sci Sports Exerc. 2016;49(2):317-26.

15. Frimeth J, Galiano E, Webster D. Some physical and clinical factors influencing the measurement of precision error, least significant change, and bone mineral density in dual-energy $\mathrm{X}$-ray absorptiometry. J Clin Densitom. 2010;13(1):29-35.

16. Hind K, Slater G, Oldroyd B, Less M, Thurlow S, Barlow M, et al. Interpretation of Dual-Energy X-Ray Absorptiometry-Derived Body Composition Change in Athletes: A Review and Recommendations for Best Practice. J Clin Densitom. 2018;21(3):429-43.

17. ISCD. ISCD Bone Densitometry Precision Calculating Tool. ISCD The internacional Society For Clinical Densitometry; 2019 [Accessed November 7, 2019]. Avaiable in: https://www.iscd.org/resources/ calculators/precision-calculator/

18. Platen P, Chae E, Antz R, Lehmann R, Kühlmorgen J, Allolio B. Bone mineral density in top level male athletes of different sports. Eur J Sport Sci. 2001;1(5):1-15.

19. Moher D, Liberati A, Tetzlaff J, Altman DG, Group P. Preferred reporting items for systematic reviews and meta-analyses: the PRISMA statement. Int J Surg. 2010;8(5):336-41.

20. Moraes, Mikael. COMPARISON OF BONE PARAMETERS BY BODY REGION IN UNIVERSITY ATHLETES: SYSTEMATIC REVIEW. versão 27 ago. 2021. SciELO Data. https://doi.org/10.48331/scielodata.R9NN4H.

21. National Heart, Lung, and Blood Institute. Study Quality Assessment Tools | National Heart, Lung and Blood Institute (NHLBI), 2019 [Accessed June 16, 2019]. Avaiable in: https://www.nhlbi.nih.gov/ health-topics/study-quality-assessment-tools

22. Kent S, Fusco F, Gray A, Jebb SA, Cairns BJ, Mihaylova B. Body mass index and healthcare costs: systematic literature review of individual participant data studies. Obes Rev. 2017;18(8):869-79.

23. Fehling PC, Alekel L, Clasey J, Rector A, Stillman RJ. A comparison of bone mineral densities among female athletes in impact loading and active loading sports. Bone. 1995;17(3):205-10.

24. Lee EJ, Long KA, Risser WL, Poindexter HB, Gibbons WE, Goldzieher J. Variations in bone status of contralateral and regional sites in young athletic women. Med Sci Sports Exerc. 1995;27(10):1354-61.

25. Nichols DL, Sanborn CF, Bonnick SL, Gench B, DiMarco N. Relationship of regional body composition to bone mineral density in college females. Med Sci Sports Exerc. 1995;27(2):178-82.

26. Taaffe DR, Snow-Harter C, Connolly DA, Robinson TL, Brown MD, Marcus R. Differential effects of swimming versus weight-bearing activity on bone mineral status of eumenorrheic athletes. J Bone Miner Res. 1995;10(4):586-93.

27. Matsumoto T, Nakagawa S, Nishida S, Hirota R. Bone density and bone metabolic markers in active collegiate athletes: findings in long-distance runners, judoists, and swimmers. Int J Sports Med. 1997;28(06):408-12

28. Taaffe DR, Robinson TL, Snow CM, Marcus R. High-impact exercise promotes bone gain in well-trained female athletes. J Bone Miner Res. 1997;12(2):255-60

29. Emslander HC, Sinaki M, Muhs JM, Chao EY, Wahner HW, Bryant SC, et al. Bone mass and muscle strength in female college athletes (runners and swimmers). Mayo Clin Proc. 1998;73(12):1151-60.

30. Rourke KM, Bowering J, Turkki P, Buckenmeyer PJ, Thomas FD, Keller BA, et al. Bone mineral density in weight-bearing and nonweight-bearing female athletes. Pediatr Exerc Sci. 1998;10(1):28-37.

31. Bemben DA, Buchanan TD, Bemben MG, Knehans AW. Influence of Type of Mechanical Loading, Menstrua Status, and Training Season on Bone Density in Young Athletes. J Strength Cond Res. 2004;18(2):220-6
32. Beals KA, Hill AK. The prevalence of disordered eating, menstrual dysfunction, and low bone minera density among US collegiate athletes. Int J Sport Nutr Exerc Metab. 2006;16(1):1-23.

33. Mudd LM, Fornetti W, Pivarnik JM. Bone mineral density in collegiate female athletes: Comparisons among sports. J Athl Train. 2007;42(3):403-8.

34. Carbuhn AF, Fernandez TE, Bragg AF, Green JS, Crouse SF. Sport and training influence bone and body composition in women collegiate athletes. J Strength Cond Res. 2010;24(7):1710-7.

35. Ackerman KE, Skrinar GS, Medvedova E, Misra M, Miller KK. Estradiol levels predict bone mineral density in male collegiate athletes: A pilot study. Clin Endocrinol (Oxf). 2012;76(3):339-45.

36. Hirsch KR, Smith-Ryan AE, Trexler ET, Roelofs EJ. Body composition and muscle characteristics of division i track and field athletes. J Strength Cond Res. 2016;30(5):1231-8.

37. Stanforth D, Lu T, Stults-Kolehmainen MA, Crim BN, Stanforth PR. Bone mineral content and density among female NCAA division 1 athletes across the competitive season and over a multi-year time frame. J Strength Cond Res. 2016;30(10):2828-38

38. Nepocatych S, Balilionis G, O'Neal EK. Analysis of dietary intake and body composition of female athletes over a competitive season. Monten J Sports Sci Med. 2017;6(2):57-65.

39. Scerpella JJ, Buehring B, Hetzel SJ, Heiderscheit BC. Increased leg bone mineral density and content during the initial years of college sport. J Strength Cond Res. 2018;32(4):1123-30.

40. Sanfilippo J, Krueger D, Heiderscheit B, Binkley N. Dual-Energy X-Ray Absorptiometry Body Composition in NCAA Division I Athletes: Exploration of Mass Distribution. Sports Health. 2019;11(5):453-60

41. Fields JB, Gallo S, Worswick JM, Busteed DR, Jones MT. 25-Hydroxyvitamin D, vitamin D binding protein, bioavailable 25 -Hydroxyvitamin $\mathrm{D}$, and body composition in a diverse sample of women collegiate Indoor Athletes. J Funct Morphol Kinesiol. 2020;5(32).

42. Taaffe DR, Marcus R. Regional and total body bone mineral density in elite collegiate male swimmers J Sports Med Phys Fitness. 1999;39(2):154-9.

43. Bazzocchi A, Ponti F, Albisinni U, Battista G, Guglielmi G. DXA: Technical aspects and application. Eur J Radiol. 2016;8(85):1481-92

44. Zymbal V, Baptista F, Fernandes P, Janz KF. Determining Skeletal Geometry. In: Biomarkers in Bone Disease; 2017. p. 621-45

45. BeckTJ. Extending DXA beyond bone mineral density: understanding hip structure analysis. Cur Osteoporos Rep. 2007;5(2):49-55.

46. Yoshikawa T, Turner CH, Peacock M, Slemenda CW, Weaver CM, Teegarden D et al. Geometric structure of the femoral neck measured using dual-energy X-ray absorptiometry. J Bone Miner Res. 1994;9(7):1053-64

47. Clarke B. Normal bone anatomy and physiology. Clin J Am Soc Nephrol. 2008;3(Supplement 3):S131-9.

48. Shepherd JA, Schousboe JT, Broy SB, Engelke K, Leslie WD. Executive Summary of the 2015 ISCD Position Development Conference on Advanced Measures From DXA and QCT: Fracture Prediction Beyond BMD. J Clin Densitom. 2015;3(18):274-86

49. Nana A, Slater GJ, Hopkins WG, Burke LM. Techniques for undertaking dual-energy X-ray absorptiometry whole-body scans to estimate body composition in tall and/or broad subjects. Int J Sport Nutr Exerc Metab. 2012;22(5):313-22.

50. Nana A, Slater GJ, Stewart AD, Burke LM. Methodology review: using dual-energy X-ray absorptiometry (DXA) for the assessment of body composition in athletes and active people. Int I Sport Nutr Exerc Metab. 2015;25(2):198-215.

51.Zemski AJ, Hind K, Keating SE, Broad EM, Marsh DJ, Slater GJ. Same-Day Vs Consecutive-Day Precision Error of Dual-Energy X-Ray Absorptiometry for Interpreting Body Composition Change in Resistance-Trained Athletes. J Clin Densitom. 2019;22(1):104-14.

52. Kosar SN. Associations of lean and fat mass measures with whole body bone mineral content and bone mineral density in female adolescent weightlifters and swimmers. Turk J Pediatr. 2016;58(1):79.

53. Singhal V, Maffazioli GD, Sokoloff NC, Ackerman KE, Lee H, Gupta N, et al. Regional fat depots and their relationship to bone density and microarchitecture in young oligo-amenorrheic athletes. Bone. 2015:(77):83-90.

54. Nevill AM, Holder RL, Maffulli N, Cheng JCY, Leung SSF, LeeWTK, et al. Adjusting bone mass for difference in projected bone area and other confounding variables: an allometric perspective. J Bone Miner Res. 2002;17(4):703-8 